\title{
Multifocal endometriosis
}

\author{
Ludmila Lozneanu ${ }^{1,2}$, Delia Gabriela Ciobanu-Apostol ${ }^{1,2}$, Raluca Anca Balan ${ }^{1}$, Ioana \\ Păvăleanu ${ }^{1}$, Simona Eliza Giuşcă ${ }^{1}$, Irina-Draga Căruntu ${ }^{1}$, Cornelia Amalinei ${ }^{*}{ }^{1,1,3}$
}

${ }^{1}$ Department of Morfofunctional Sciences I, "Grigore T. Popa" University of Medicine and Pharmacy, Iasi, Romania, ${ }^{2}$ Department of Pathology, "Sf. Spiridon" Clinical Emergency Hospital, lasi, Romania, ${ }^{3}$ Department of Pathology, Institute of Forensic Medicine, lasi, Romania

\begin{abstract}
Endometriosis represents a complex entity, characterized by tissue located in ectopic sites, outside uterus, with the highest incidence in ovaries and peritoneum. We report a rare case, with multiple endometriotic foci, in a 34 year old woman. The patient presented with intestinal subocclusion syndrome, associated with pelvic and abdominal pain. The imagistic exploration identified two peritoneal and one left ovarian masses and the clinical exam detected a 48/22 mm Douglas pouch mass. The surgical treatment, by exploratory laparotomy, resulted in segmental resection of the rectosigmoid junction and left adnexectomy. The intraoperative frozen section consultation, followed by paraffin-embedded, routine and immunohistochemical staining, led to a rare diagnosis of multiple foci of endometriosis, involving left adnexa, colorectum, and pericolic lymph nodes, without any atypia or malignancy. The postoperative evolution had been favorable. According to the identification of multiple endometriotic foci in our case, the hypothesis of different etiopathogenic mechanisms involvement may be supported by our findings.
\end{abstract}

Keywords: endometriosis, multiple foci, immunohistochemistry

\section{Introduction}

Endometriosis concept is reflecting a pathologic process characterized by the presence of endometrial glands and stroma in an extra-uterine location, most frequently associated with chronic inflammatory reaction. Endometriosis involves $10-15 \%$ of women of reproductive age, being stimulated by ovarian hormones [1]. The high association of endometriosis with infertility triggered numerous related studies. Despite scientists' interest, the origin and the mechanism of endometriosis development are still incompletely deciphered. There are several

Received: July 2016; Accepted after review: September 2016; Published: September 2016.

${ }^{*}$ Corresponding author: Prof. Cornelia Amalinei, MD, PhD, "Grigore T. Popa" University of Medicine and Pharmacy lasi; Institute of Forensic Medicine, 16 Universitatii Street, Iasi-700115, Romania

E-mail: cornelia.amalinei@umfiasi.ro theories which interconnect several mechanisms as attempts to elucidate the initiation pathways and the treatment approach in endometriosis. Although the most frequent location is pelvis (ovary, peritoneum), we present a multiple location (adnexa, colon, and lymph nodes), as a rare diagnostic entity. Within this context, the characteristics of our case may support several associated etiopathogenic mechanisms and developmental pathways in endometriosis.

\section{Case report}

We are reporting the case of a 34 year old patient, admitted in Department of Surgery, First Surgery Clinic, "Sf. Spiridon" Emergency Hospital, Iasi, Romania, in January 2016, with subocclusive syndrome, colicative pelvic and abdominal pain, and rectorrhagia. The patient declared a history of decreased bowel movements, with onset one year prior to 
hospitalization, without any family gynecological or endometriosis history. The preoperative assessment by imagistic studies (ultrasound, abdominal and pelvic CT scans) revealed three tumor masses, two of them located in the peritoneum and another one in the left ovary, highly suspicious of malignancy. Clinical examination also found a 48/22 mm mass in Douglas pouch, between the uterus and the sigmoid colon. Following these observations, exploratory laparotomy has been performed, with left adnexectomy and segmental resection of rectosigmoid junction, considered as necessary due to deep, profound infiltration and severe symptomatology. Intra-operative frozen section consultation has been requested for the adnexal mass.

Pathologic examination has been performed in the Department of Pathology, "Sf. Spiridon" Emergency Hospital, lasi, Romania. The intra-operative frozen section consultation of the left adnexa showed endometrial glands and stroma distributed within a vascular connective tissue, features highly suggestive of endometriosis. The gross inspection of the rectosigmoid junction showed the presence of an ulcerovegetant mass, measuring $5 / 3.5 / 1.6 \mathrm{~cm}$, with heterogeneous features, white-tan color, with focal hemorrhage, and increased consistency. Grossly, five pericolic lymph nodes have been identified. A hemorrhagic cystic lesion of approximately $32 \mathrm{~mm}$ has been noticed in the ovary, with brown-violet color, smooth inner wall, and evident vascularization. The surgical specimens have been paraffin-embedded, followed by $4 \mu \mathrm{m}$ microtome sections and routine Hematoxylin-eosin staining.

Microscopy showed the presence of multiple foci of endometriosis, composed of endometrial glands and stroma, with left paratubal and cortical ovarian location (Figures $1 \mathrm{a}$ and 1b), transmural rectosigmoidian involvement, with submucosal, muscularis propria (Figures 2a and $2 \mathrm{~b}$ ), and serosal invasion (Figure 3 ), and two subcapsular lymph nodes implants (Figure 4). The glands have been lined by simple columnar epithelium of endometrial type, without evidence of significant proliferative activity or atypia, associated with local inflammatory reaction. The lesion has been typically associated with hemosiderin laden macrophages and perilesional fibrosis. In order to confirm the presence of endometriosis areas, immunohistochemistry has been performed in the Department of Pathology, using several markers, as following: CD10 and Vimentin have been mainly used for the stromal component (Figures $5 a$ and $5 b$ ) and hormonal receptors, ER (estrogen receptor) and PR (progesterone receptor), (Figures $6 \mathrm{a}$ and $6 b$ ) have been mainly used for the glandular component. The morphological features identified corroborated with the immunohistochemical findings have set the diagnosis of multifocal endometriosis.

The postoperative evolution had been favorable, without any complications and routine follow up had been decided.

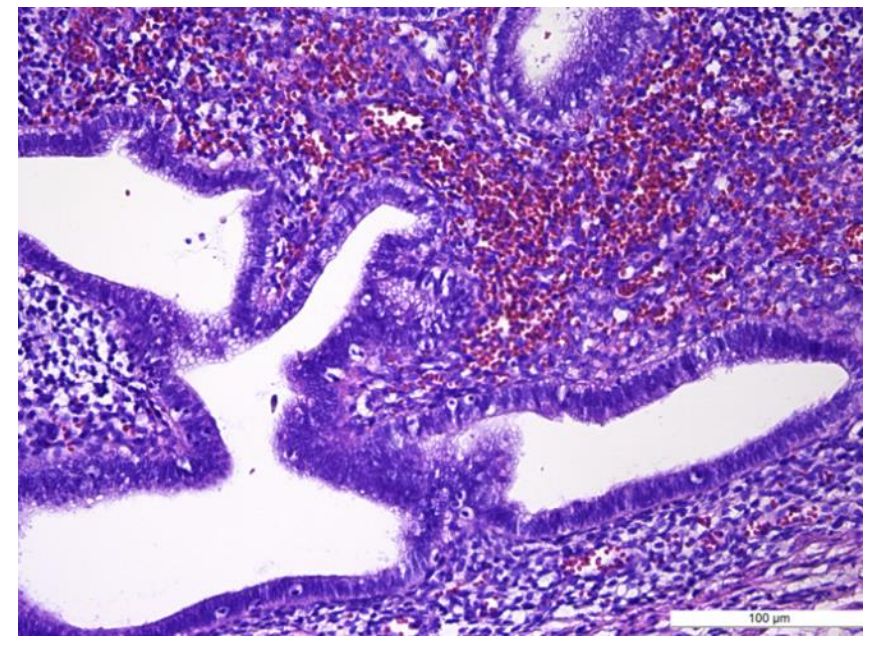

Fig. 1a. Left mesosalpinx endometriotic focus (HE, x200) 


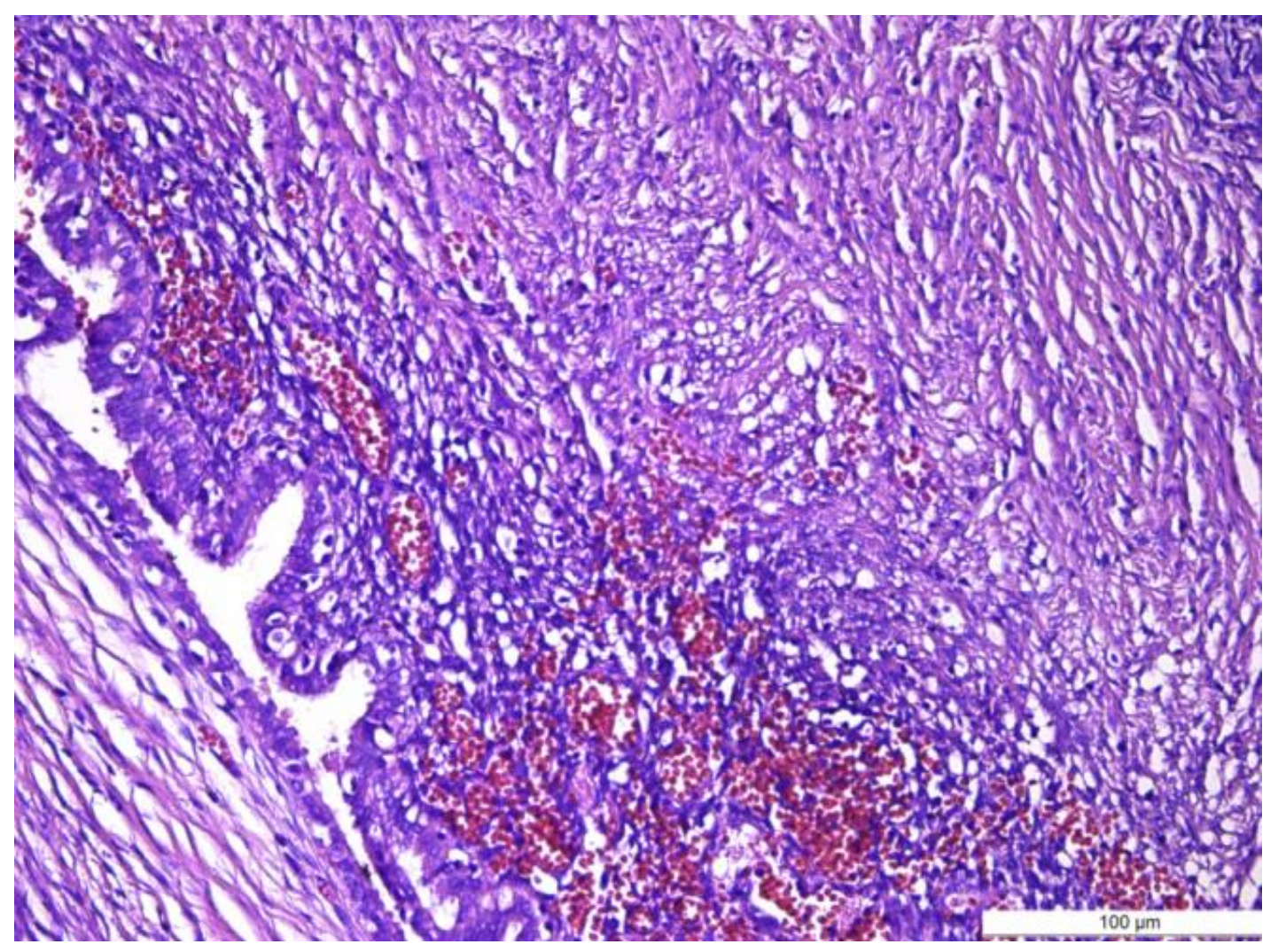

Fig. 1b. Left ovary endometriotic focus (HE, x200)

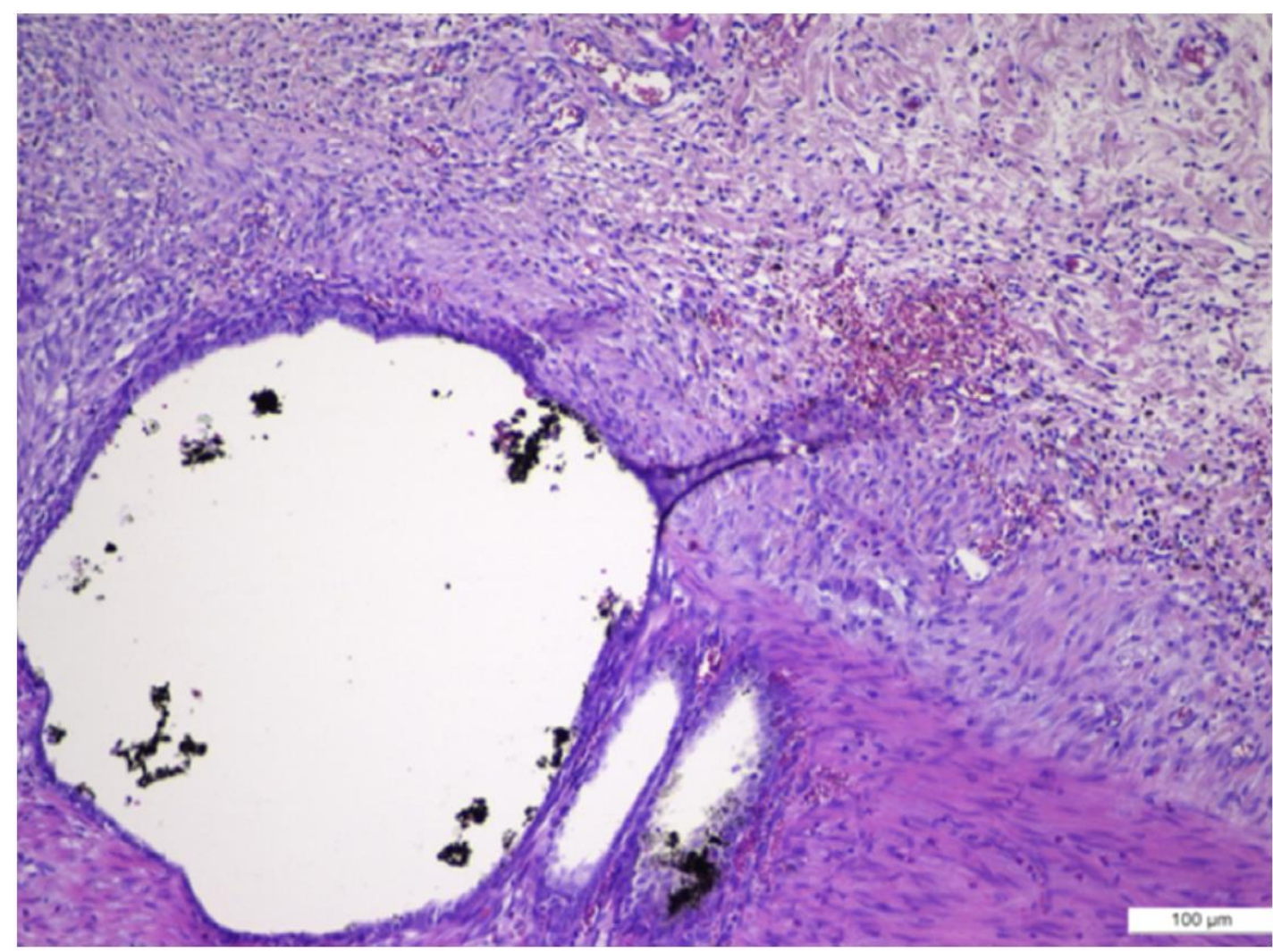

Fig. 2a. Colonic submucosal endometriotic focus (HE, x100) 


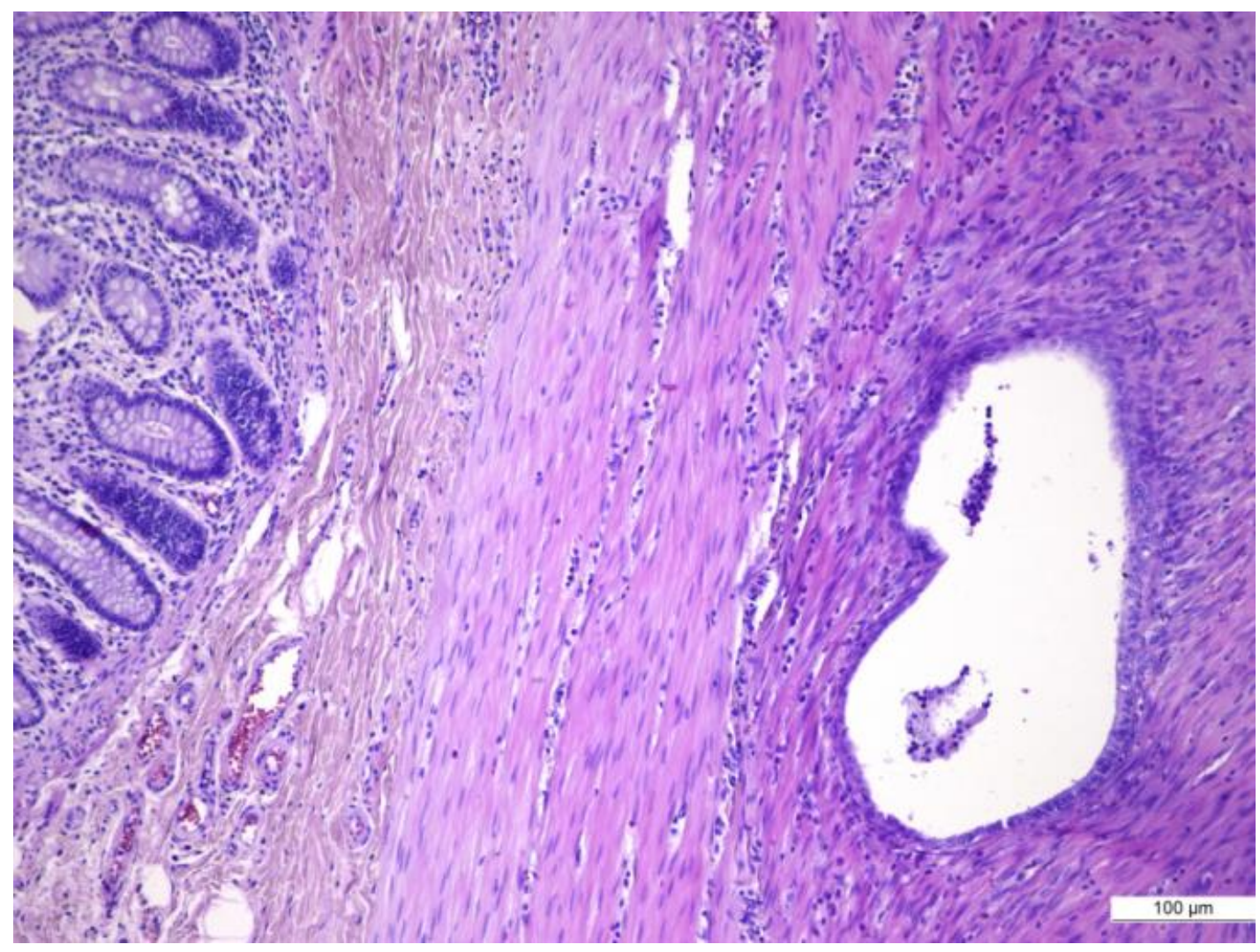

Fig. 2b. Colonic muscularis propria endometriotic focus (HE, x100)

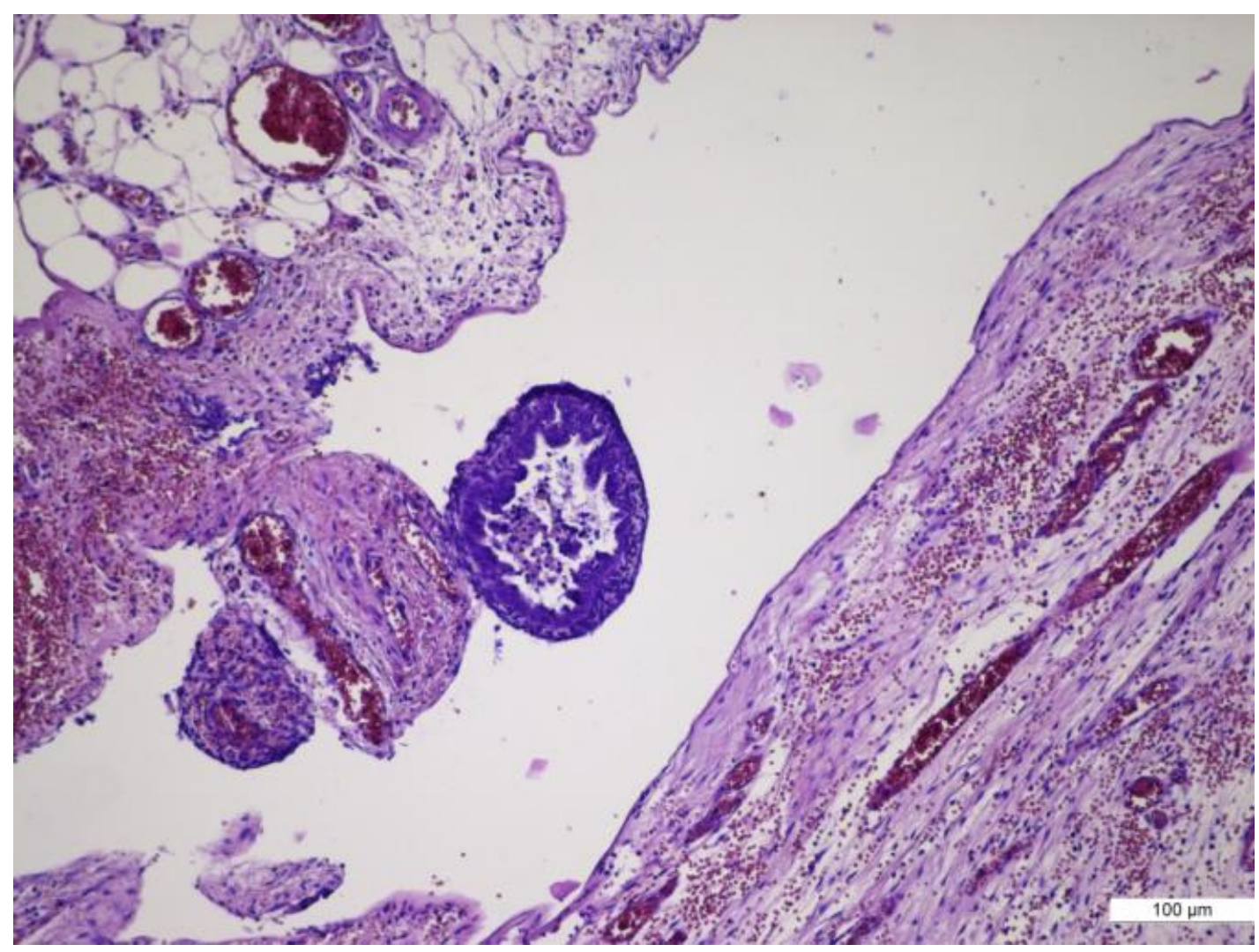

Fig. 3. Endometriotic focus exhibiting a single gland adherent to the peritoneal serosa (HE, $x 100)$ 


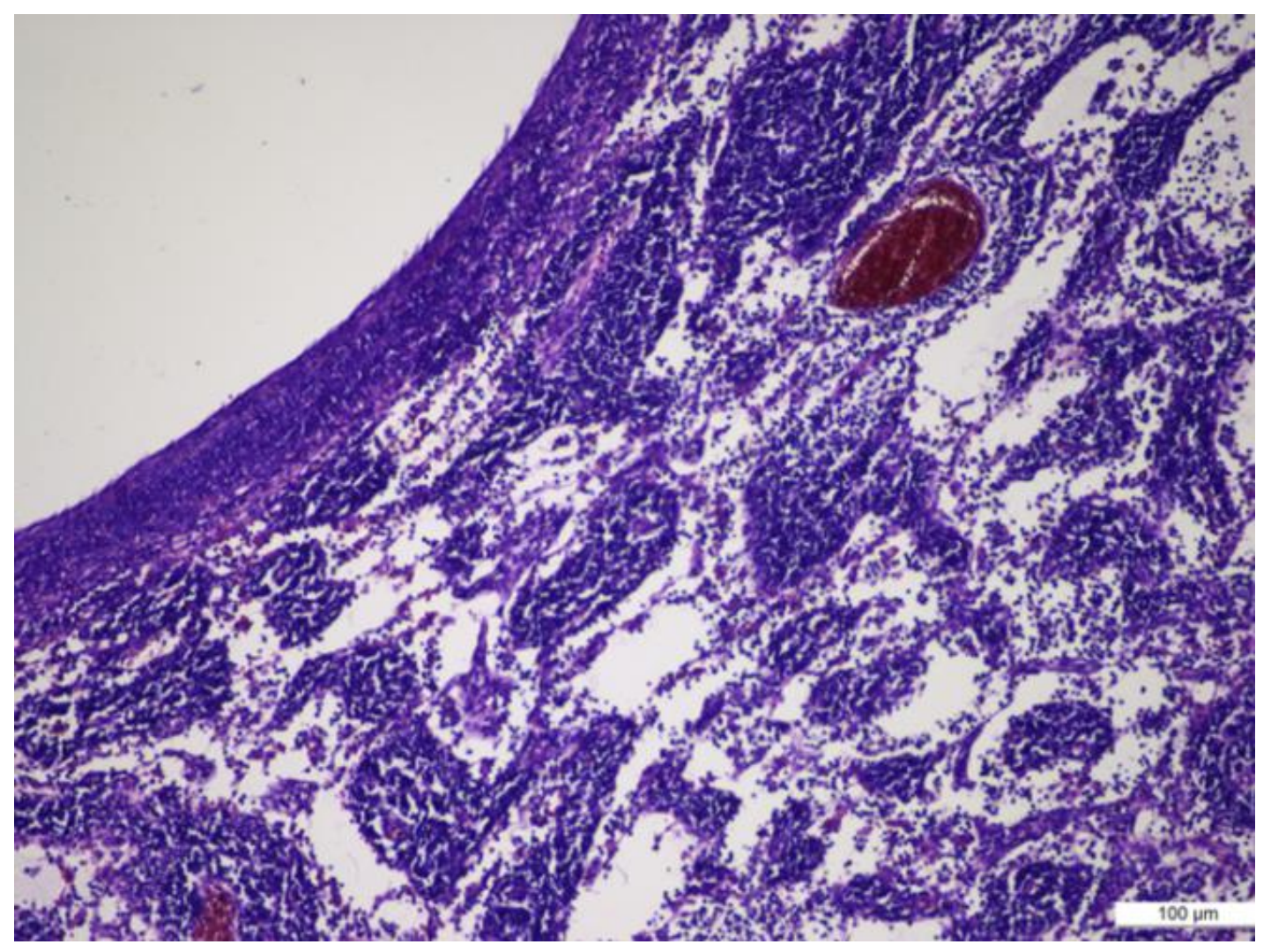

Fig. 4. Lymph node endometriosis (HE, x100)

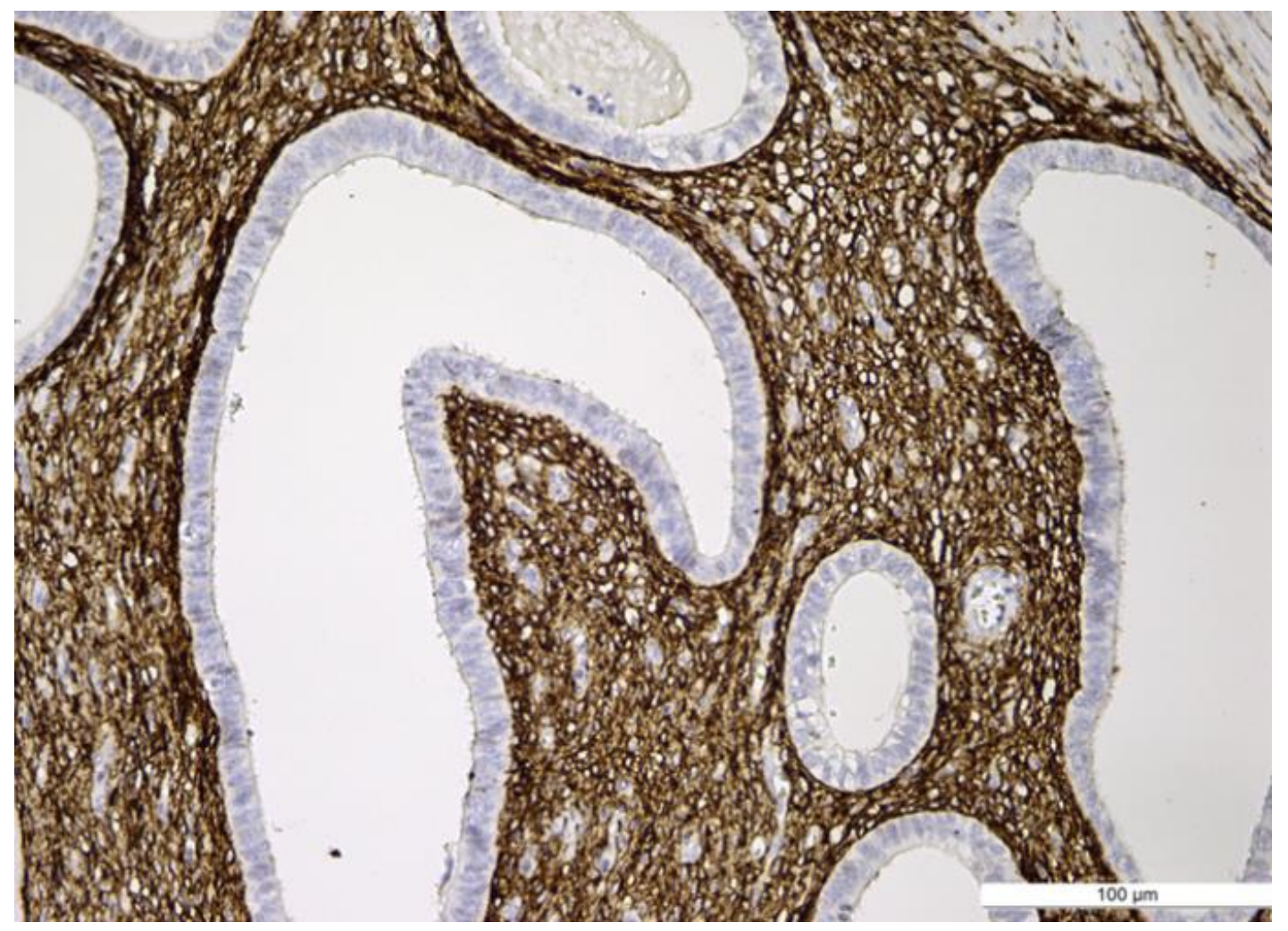

Fig. 5a. CD10 positive in stromal cells of a colonic endometriotic focus (IHC, Ab anti-CD10, x400) 


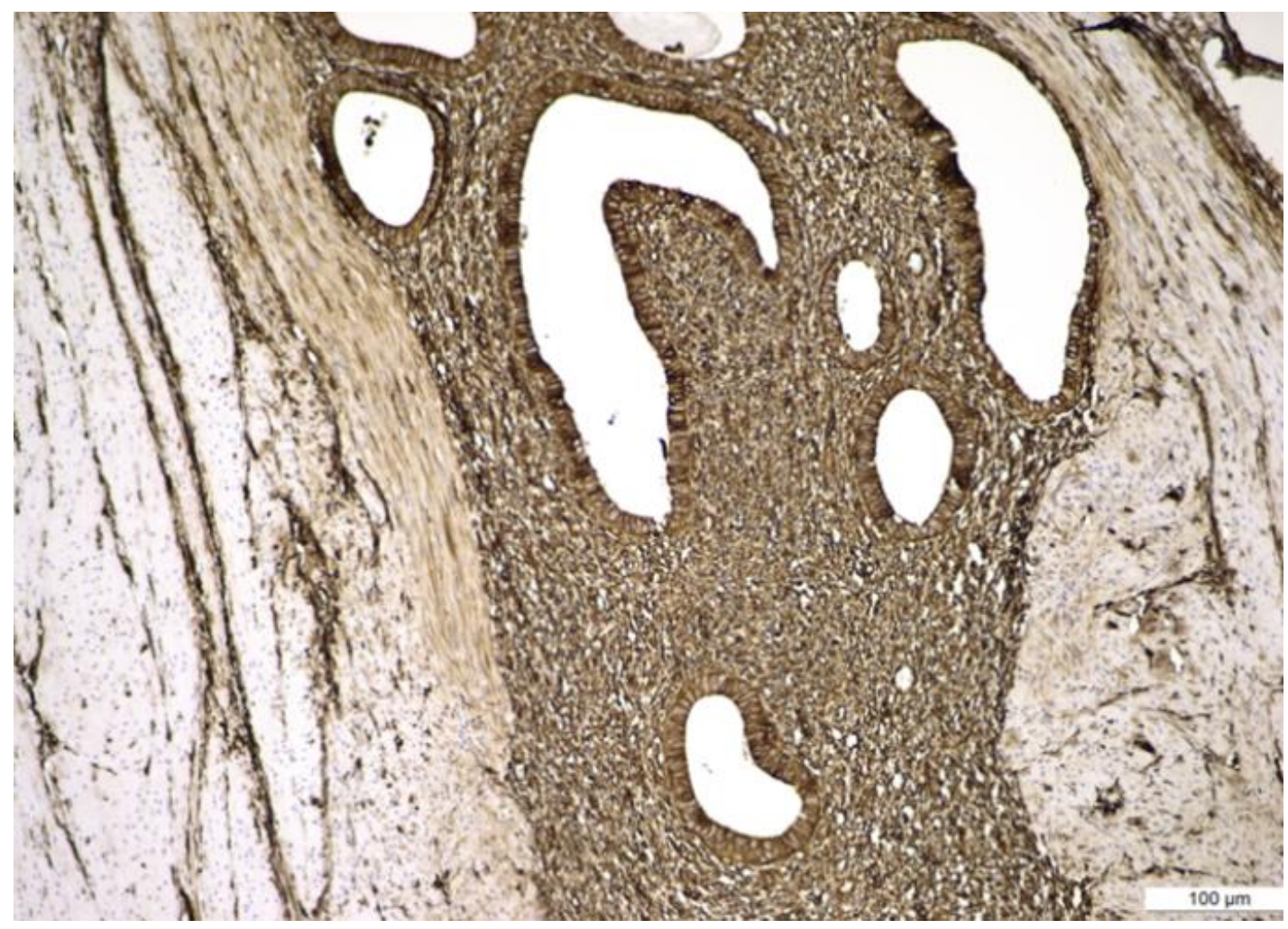

Fig. 5b. Stroma and glandular components of a colonic endometriotic focus (IHC, Ab anti-Vimentin, x100)

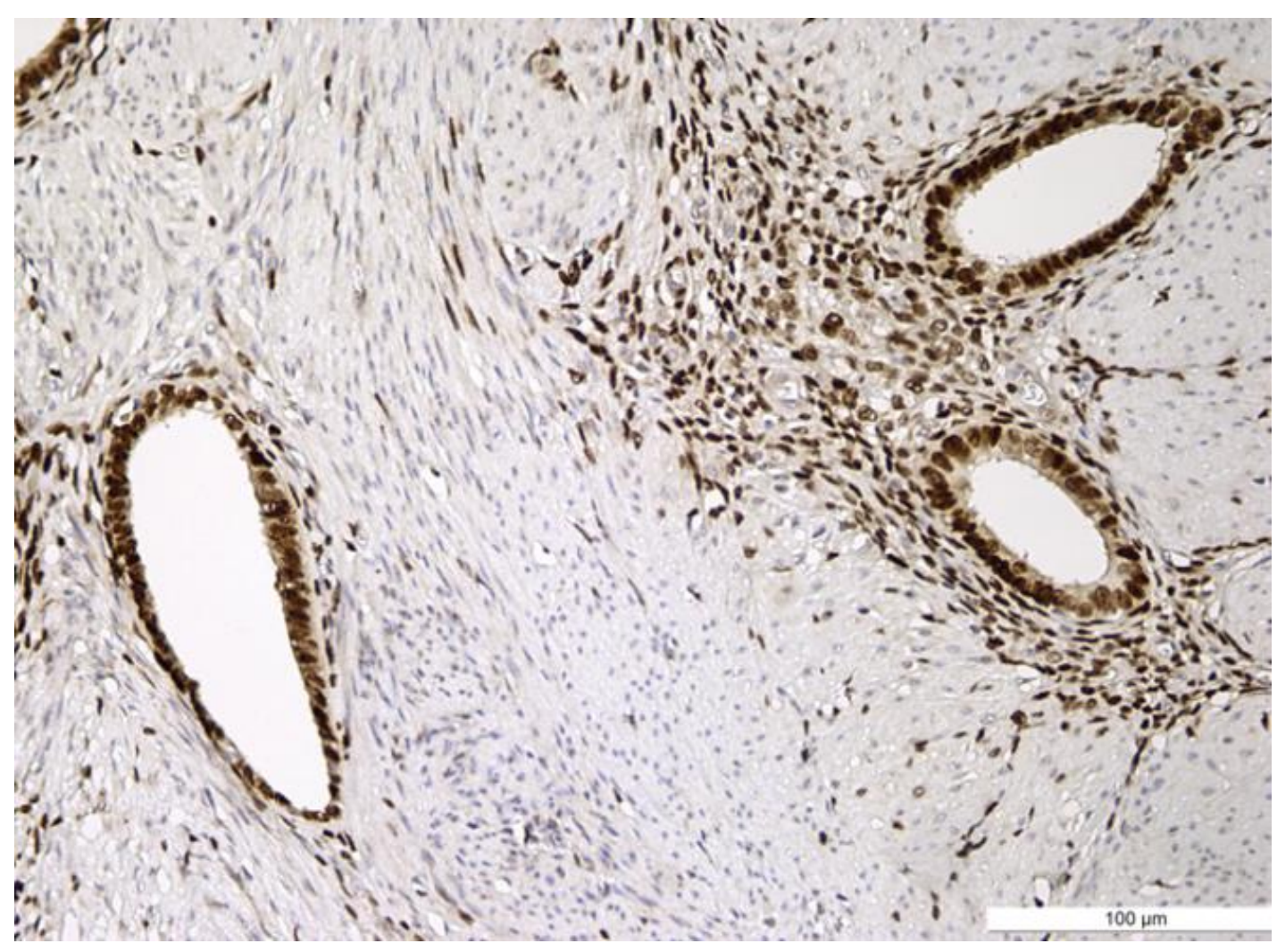

Fig. 6a. Glandular and stromal components of a colonic endometriotic focus (IHC, Ab anti-ER, x200) 


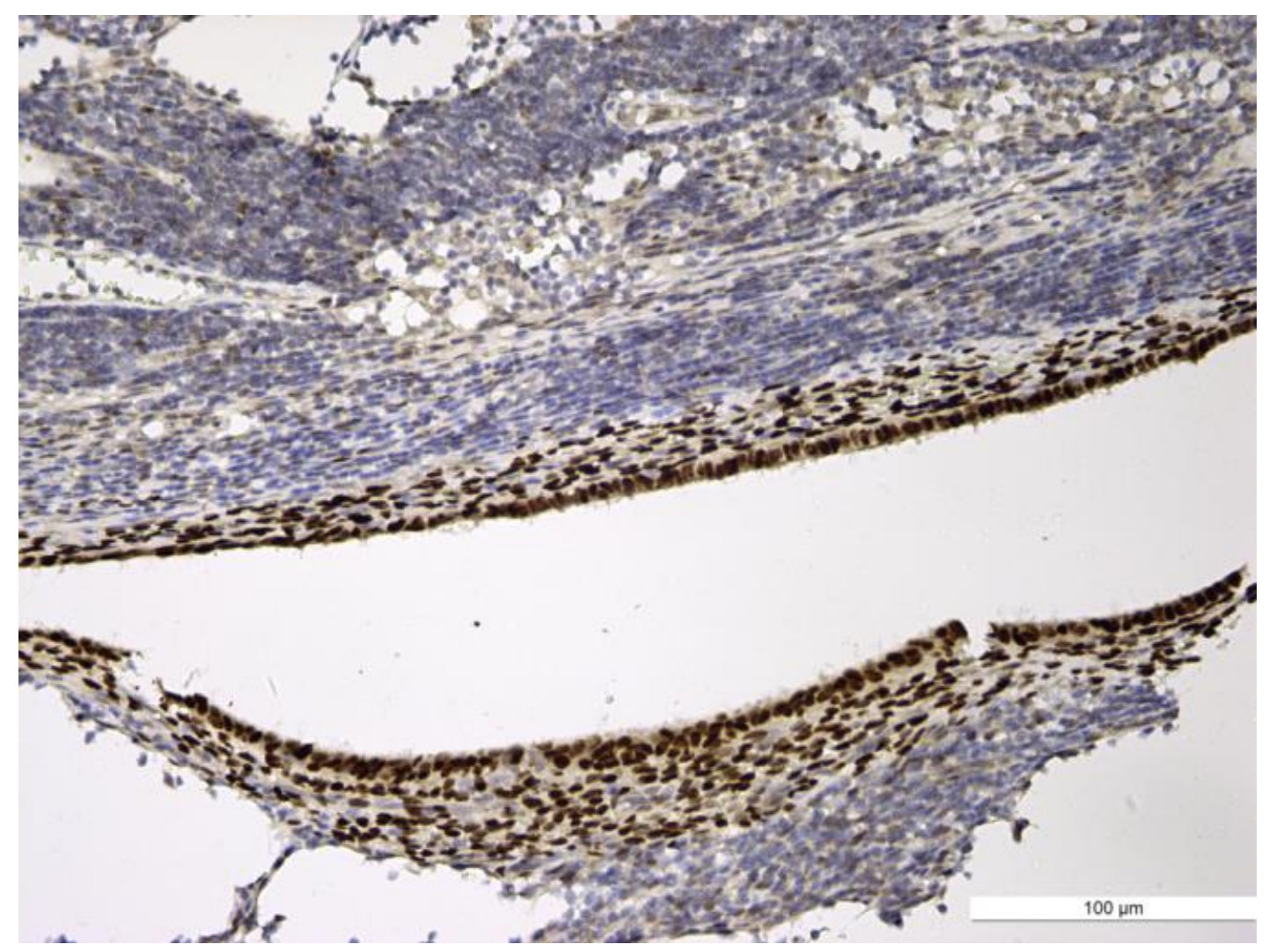

Fig. 6b. Glandular and stromal components of lymph node endometriosis (IHC, Ab anti-PR, x200)

\section{Discussions}

Endometriosis involves approximately $2 \%$ of the general female population [2] and approximately $70 \%$ of these cases have associated pelvic inflammatory diseases [3]. The exact prevalence is still unknown due to asymptomatic cases and incidental identification of the disease. Endometriosis is associated with infertility in $25-30 \%$ cases [4]. Endometriosis consists of a complex lesion, with chronic evolution, mainly diagnosed during reproductive life, as had been also the patients' age (34 years old), in the presented case, although in our last year files we registered a broad age distribution (25-74 years old). Endometriosis is rare after menopause, a characteristic that certifies the estrogenic dependence [4], being diagnosed in only $12.50 \%$ of cases in post-menopause, in our last year files. Deep endometriosis is frequently the reason for patients' presentation for a gynecological consultation due to infertility, dyspareunia, dysmenorrhea, and chronic pelvic pain [5], the latter symptom being the clinical manifestation in our patient.
Endometriosis can be abdominal and extra-abdominal [3]. The abdominal type may be intraperitoneal (ovarian, broad ligament, tubal, pelvic peritoneum, Douglas space, appendix, and small intestine) or extraperitoneal (cervix, vagina, recto-vaginal septum, round ligament, and post-surgery abdominal scars, mainly post C-section) $[3,6]$. The locations from our last year files had been, in descending order of frequency: ovary $(40 \%)$, uterine cervix $(20 \%)$, post-C section (13.33\%), appendix (13.33\%), colon (6.66\%), fallopian tube $(6.66 \%)$, the symptoms being variable, from intestinal occlusion, acute appendicitis, cicatricial external endometriosis, pelvic pain accentuated during menstruation, and colonic motility disorders. The presented case is the only case from our files with multiple locations: left adnexa, rectosigmoid, and pericolic lymph nodes.

Extra-abdominal endometriosis is rare, such as hepatic [7], pulmonary [8], urinary tract [9], cutaneous, and nasal cavity [3]. Other rare locations reported in literature are: iliac vein wall [10] and hernial sac wall [11]. 
Gastro-intestinal endometriosis is frequently reported in colorectum $(70 \%)$, as had also been in two cases form our files, including the presented patient, and ileum (1$7 \%)[12,13]$. Rectosigmoid and lymph node endometriosis is reported in $26.3 \%$ to $50 \%$ of cases [14, 15], being confirmed in several studies [6, 13-20]. Considering the literature data, the possibility of ectopic implants to be independently produced by retrograde menstruation to three different sites, or the participation of a metaplastic process involved in colonic location, associated with the possibility of dissemination, by lymphatics, from ovary and, furthermore, from colon to lymph nodes, are probable mechanisms. The degree of contribution of each mechanism to the complex picture of this case remains speculative.

The risk of lymph node endometriosis is increased when intestinal endometriosis is circumferential, as has been also noticed in the presented case (transmural involvement), and over $1.75 \mathrm{~cm}$ length [14], as has been also measured in our patient $(5 / 3.5 / 1.6 \mathrm{~cm})$. According to our review, this is the only involvement of two lymph nodes in our files.

Numerous theories and etiopathogenic mechanisms are debated in literature. Although the most plausible hypothesis is that of retrograde menstruation, especially in cases with adnexal location, according to Sampson's theory [21], other hypotheses seem to be more likely involved in other locations. In colon endometriosis, caelomic epithelium metaplasia is the most plausible phenomena, according to Meyer's theory [22], and lymphatic dissemination seems to be more likely involved in lymph nodes endometriosis, as stated by Halban's theory [23]. According to literature data, the mechanism of lymph node involvement is still unclear. In order to explain the significance of lymph node involvement, several theories have been launched. According to the some opinions, lymph node involvement may signify a chronic intestinal endometriosis [24]. Some authors support the low incidence of lymph node involvement in ovarian and/or peritoneal disease [25]. The identification of endometrial elements in lymph nodes may be highly correlated to the coexistence of possible foci of endometriosis encompassing the evident lesions, as a strong predictor of local or distance recurrence [13]. Another hypothesis is that endometriosis histogenesis may be different according to the location and lesion type [26]. Thus, ovarian and deep endometriosis may probably originate from a metaplastic process, while peritoneal lesions may probably be the result of peritoneal implantation [26]. A relatively recent theory is that of Müllerian remnants or stem cells activation in this location [27]. Considering the multiple foci of endometriosis in the presented case, the mechanism of retrograde menstruation (for the ovarian location) could be associated with a metaplastic process of the caelomic epithelium (for the colonic location) and a lymphatic dissemination (for the pericolic lymph node location).

Endometriosis is a highly heterogeneous entity, considering its hormonal, immunological, genetic, metabolic influences, and characteristics of a systemic disease, with typical and atypical features manifested during the disease and evolution toward adherences, invasion, and metastasizing [28]. Normal endometrium is different from that of a patient with endometriosis due to proliferative, apoptotic, invasive, (lymph)angiogenic, hormonal, and immunological characteristics similar to a malignant process [14, 29], with high risk of recurrences, either as consequences or of intrinsic pathogenic characteristics. There are numerous literature data to support endometriosis association with endometrioid and clear cell ovarian carcinoma [30]. No evidence of cytological atypia and no significant mitotic activity have been identified, thus the malignant transformation has been excluded in the presented case.

The differential diagnosis included other clinical instances that could result in pelvic and abdominal pain, such as rectorrhagia, constipation, peritoneal proliferation, and pelvic disorders, or neuromuscular and musculo-skeletal chronic lesions [31].

The morphological differential diagnosis had to be done with ovarian endosalpingiosis [32], a lesion frequently overdiagnosed as endometriosis, due to histological resemblance, and ovarian serous cystic lesions, while colon location has been 
differentiated from colon carcinoma and lymph nodes from cystic lesions, all these being excluded by characteristic histology. Supplementary, immunohistochemical profile performed in the presented case has confirmed the presence of areas of endometriosis by $\mathrm{ER}$ and $\mathrm{PR}$ dominant epithelial, CD10 stromal, and Vimentin stromal and epithelial immunopositivity

\section{Conclusions}

The presented case illustrates a typical endometriosis, diagnosed during the reproductive age, asymptomatically developed until the onset of a subocclusive syndrome. Considering the particularities of this case, although relatively rare, the possibility of endometriosis occurrence and its differential diagnosis should be considered in young women with abdominal pathology. Due to multiple foci identified in the presented case,

\section{References}

1. Nothnick W, Alali Z. Recent advances in the understanding of endometriosis: the role of inflammatory mediators in disease pathogenesis and treatment. F1000 Res 2016; 5:F1000 Faculty Rev 186.

2. Machairiotis N, Stylianaki A, Dryllis G, et al. Extrapelvic endometriosis: a rare entity or an under diagnosed condition? Diagn Pathol 2013; 8:194.

3. De Ceglie A, Bilardi C, Blanchi S, et al. Acute small bowel obstruction caused by endometriosis: a case report and review of the literature. World J Gastroenterol 2008; 14:34303434.

4. Bulletti C, Coccia ME, Battistoni S, Borini A. Endometriosis and infertility. J Assist Reprod Genet 2010; 27:441-447.

5. Ruffo G, Scopelliti F, Scioscia M, Ceccaroni M, Mainardi P, Minelli L. Laparoscopic colorectal resection for deep infiltrating endometriosis: analysis of 436 cases. Surg Endosc 2010; 24:63-67.

6. Insilla $C A$, Granai $M$, Gallippi $G$, et al. Deep endometriosis with pericolic lymph node involvement: a case report and literature review. World J Gastroenterol 2014; 20:6675-6679.

7. Fluegen G, Jankowiak $F$, Zacarias Foehrding L, Kroepil F, Knoefel WT, Topp SA. the hypothesis of combined etiopathogenic mechanisms may be supported.

Numerous research data have demonstrated that endometriosis has the characteristics of a benign proliferative process with the possibility of malignant transformation. Although the stage of "atypical" endometriosis is rarely seen in practice, the malignancy risk should be considered as an intrinsic characteristic and should be dismissed only after a thoughtful, systematical analysis of the involved tissue.

\section{Consent}

Written informed consent was obtained from the patient for publication of this case report and accompanying images.

\section{Conflict of interest}

The authors declare no competing interest.
Intrahepatic endometriosis as differential diagnosis: case report and literature review. World $J$ Gastroenterol 2013; 19:4818-4822.

8. Jablonski C, Alifano M, Regnard JF, Gompel A. Pneumoperitoneum associated with catamenial pneumothorax in women with thoracic endometriosis. Fertil Steril 2009; 91(3):930.

9. Cheng $\mathrm{CH}$, Kuo HC, Su B. Endometriosis in a kidney with focal xanthogranulomatous pyelonephritis and a perinephric abscess. BMC Res Notes 2015; 8:591.

10. Zamurovic M. Rare extrapelvic endometriosis on iliac vein wall-diagnosis and treatment. Clin Exp Obstet Gynecol 2014; 41:349450 .

11. Albutt K, Glass C, Odom S, Gupta A. Endometriosis within a left-sided inguinal hernia sac. J Surg Case Rep 2014; 2014(5).

12. Mechsner $S$, Weichbrodt $M$, Riedlinger $W F$, et al. Estrogen and progestogen receptor positive endometriotic lesions and disseminated cells in pelvic sentinel lymph nodes of patients with deep infiltrating rectovaginal endometriosis: a pilot study. Hum Reprod 2008; 23:2202-2209.

13. Mechsner $S$, Weichbrodt $M$, Riedlinger WF, Kaufmann AM, Schneider A, Köhler C. Immunohistochemical evaluation of endometriotic lesions and disseminated endometriosis-like cells in 
incidental lymph nodes of patients with endometriosis. Fertil Steril 2010; 94:457-463.

14. Abrao MS, Podgaec S, Dias JA Jr, et al. Deeply infiltrating endometriosis affecting the rectum and lymph nodes. Fertil Steril 2006; 86:543547.

15. Noel JC, Chapron C, Fayt I, Anaf V. Lymph node involvement and lymphovascular invasion in deep infiltrating rectosigmoid endometriosis. Fertil Steril 2008; 89:1069-1072.

16. Jerby BL, Kessler $H$, Falcone $T$, Milsom JW. Laparoscopic management of colorectal endometriosis. Surg Endosc 1999; 13:1125-1128.

17. Poyatos LR, Perez PA, Bravo BF, et al. Rectosigmoid endometriosis with lymph node involvement. Gastroenterol Hepatol 2003; 26:23-25.

18. Thomakos N, Rodolakis A, Vlachos $G$, Papaspirou I, Markaki S, Antsaklis A. A rare case of rectovaginal endometriosis with lymph node involvement. Gynecol Obstet Invest 2006; 62:45-47.

19. Barrier BF, Dick EJ Jr, Butler SD, Hubbard GB. Endometriosis involving the ileocaecal junction with regional lymph node involvement in the baboon-striking pathological finding identical between the human and the baboon: a case report. Hum Reprod 2007; 22:272-274.

20. Rossini R, Monsellato $D$, Bertolaccini $L$, et al. Lymph node involvement in deep infiltrating intestinal endometriosis: does it really mean anything? J Minim Invasive Gynecol 2016; 23:787792.

21. Sampson JA. Metastatic or embolic endometriosis, due to the menstrual dissemination of endometrial tissue into the venous circulation. Am J Pathol 1927; 3(2):93-110.

22. Meyer R. Über den Stand der Frage der Andenomyositis und Adenomyome im allgemeinen und insbesondere über Adenomyositis seroepithelialis und Adenomyometritis sacromatosa. Zentralb/ Gynakol 1919; 36:754.
23. Simpson JL, Bischoff FZ, Kamat A, Buster JE, Carson SA. Genetics of endometriosis. Obstet Gynecol Clin North Am 2003; 30:21-40.

24. Bulun SE. Endometriosis. $N$ Engl J Med 2009; 3:268-279.

25. Tempfer CB, Wenzl $R$, Horvat $R$, et al. Lymphatic spread of endometriosis to pelvic sentinel lymph nodes: a prospective clinical study. Fertil Steril 2011; 96:692-696.

26. Nisolle M, Donnez J. Peritoneal endometriosis, ovarian endometriosis, and adenomyotic nodules of the rectovaginal septum are three different entities. Fertil Steril 1997; 68:585-596.

27. Gargett CE, Schwab KE, Deane JA. Endometrial stem/progenitor cells: the first 10 years. Hum Reprod Update 2016; 22:137-163.

28. Jerman LF, Hey-Cunningham AJ. The role of the lymphatic system in endometriosis: a comprehensive review of the literature. Biol Reprod 2015; 92(3):64.

29. Berbic $\mathrm{M}$, Hey-Cunningham $A, \mathrm{Ng} \mathrm{C}$, et al. The role of Foxp3p regulatory T-cells in endometriosis: a potential controlling mechanism for a complex, chronic immunological condition. Hum Reprod 2010; 25:900-907.

30. Schmidt D, Ulrich U. Endometriosis-related ovarian tumors. Pathologe 2014; 35:348-354.

31. Troyer MR. Differential diagnosis of endometriosis in a young adult woman with nonspecific low back pain. Phys Ther 2007; 87:801810.

32. De Wilde RL, Hesseling M, Hirschelmann G, Tchartchian G, Verhoeven HC. Differential Diagnosis in Endometriosis: Endosalpingiosis; the Unknown Entity. An Overview of 1,100 VideoDocumented Laparoscopies. JMIG 2012; 19:S93S94. 